\title{
The First Synthesis of Dually Modified Southern-Mimicking Nucleoside: 4'-Methyl Branched and Bicyclo [3.1.0] Hexane Locked Nucleoside
}

\author{
Kwan Woo Kim and Joon Hee Hong” \\ College of Phamacy, Chosun University, Gwangin 501-759, Korea \\ Recened Januay 31, 2004
}

\begin{abstract}
This paper reports the stereoselective synthesis of a novel nucleoside. 4'-methyl branched and bicyclo [3.1.0] hexane locked-nucleoside 12, using a sequential [3,3]-sigmatropic rearrangenent carbene cycloaddition reaction/Curtius reaction strategy with a high stereoselectivity,
\end{abstract}

Key Words : Locked-nucleoside, [3,3]-Sigmatropic rearrangement, Curtius reaction, Antiviral agents

\section{Introduction}

A number of purine and pyrimidine carbocyclic nucleosides built on a rigid bicyclo[3.1.0] hexane template (Figure 1) ${ }^{\prime}$ were recently synthesized and evaluated for their potential as antitumor or antiviral agents. The rigid bicyclo[3.1.0] hexane template is a system that mimics either the northern-type (,E) or southern-type conformation $(, \mathrm{E})$, as defined in the pseudorotational cycle. ${ }^{2}$ Considering that this template mimics the active, receptor-bound conformation of either a nucleoside or nucleotide, it can identify the favored sugar conformation that results in the optimal recognition by the target enzyme. Another interesting feature of carbocyclic nucleosides is that a number of carbocyclic adenosine analogues possess antiviral activity by inhibiting $S$-adenosylhomocystenine hydrolase. ${ }^{3}$ Moreover, this mechanisın might be exploited as a combination therapy in association with the nucleosides with a different mechanism of action.

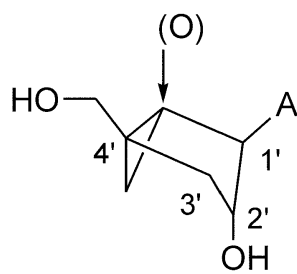

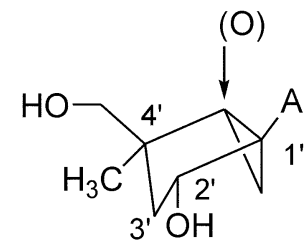

2E ('North'-like)<smiles>[B][C@@H]1C(O)C[C@@]1(CO)CCC</smiles>

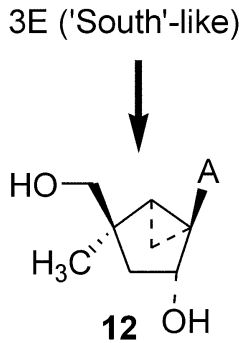

Northern-like conformation

Southern-like conformation

Figure 1. Structures of hicyelo[3.1.0]hexane template and target compound 12 .

\footnotetext{
Corresponding Author: Tel: +82-62-230-6378; Fax: +82-62-
} 222-5414; e-mail: hongih(a)chosun.ac.kr
Furthermore, the $4^{\prime} \alpha-C$ homologated furanose nucleosides, ${ }^{+}$particularly the alkyl branches, are molecules of considerable current interest. One of reasons for this interest arises from their notable biological activities as antiviral and antitumor agents, as illustrated by $4^{\prime} \alpha-C^{\prime}$-methyl-thymidine $\left(\mathrm{EC}_{50}=7.2 \mu \mathrm{M}\right.$ against $\mathrm{HIV}$ in $\mathrm{MT}-4$ cell $),{ }^{5} 4^{\prime} \alpha$-C-fluoromethyl-2'-deoxycytidine, ${ }^{6} 4^{\prime} \alpha$-C-hydroxymethylthymidine? and $4^{\prime} \alpha$ - $C$-azidomethyl-thymidine. ${ }^{8}$

With regards to these interesting mechanisms, as well as the antiviral activity of branched and locked carbocyclic nucleosides, this study synthesized and assayed a novel carbocyclic nucleoside, which is hybrid of a 4'-branched nucleoside and a bicycle[3.1.0] hexane locked nucleoside.

\section{Results and Discussions}

An $\gamma \delta$-unsaturated ester 1 was selected as the starting compound for synthesizing the target nucleosides, which was readily synthesized from acetol using a method reported elsewhere. ${ }^{9}$ As shown in Scheme 1, the ester was hydrolyzed to give the acid derivative 2 in an $82 \%$ yield. The second stage of synthesizing the bicyclo[3.1.0]hexane system from the olefinic acid 2 was begun using an intramolecular carbene cycloaddition reaction. The $\beta$-keto ester 3 was synthesized by activating the acid 2 with carbonyldiimidazole followed by a Claisen-like condensation using methyl 2-lithioacetate. Compound $\mathbf{3}$ was subjected to a diazo transfer reaction with tosyl azide in the presence of triethylamine to give the desired diazo compound 4 in an $89 \%$ yield. Unfortunately, the construction bicyclo[3.1.0]hexane system was performed using copper(II) acetylacetonate ${ }^{10}$ to produce a 1:1 mixture of the desired less polar isomer 5 $(43 \%)$ and an unwanted isomer and $5^{\prime}(42 \%)$. Each isomer 5 and 5 ' was subjected to the carbonyl reduction procedure using $\mathrm{NaBH}_{4}$ because it was too difficult to determine their stereochemistry at this stage. A thorough NOE study on the structure of the reduced product 6 (Figure 2), which relied on the reasonable convex addition of the bicyclo[3.1.0]hexane system 5 , indicated that the stereochemical assignment of the bicycle[3.1.0] hexanes 5 and 5 ' was correct. 'The single stereochemical outcome 6 from 5 could readily be explained in terms of the convex nucleophilic addition of the 

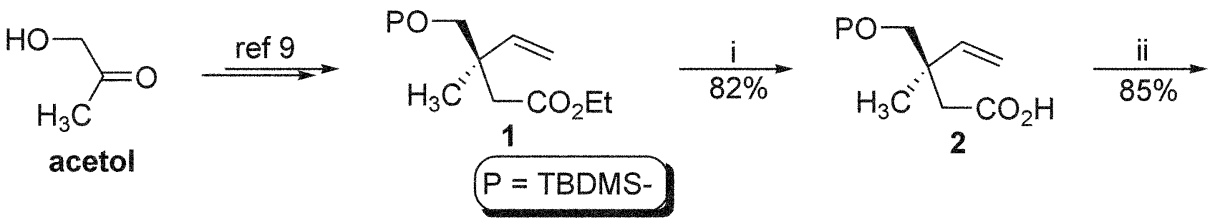<smiles>C=C[C@](C)(CC(=O)O)CC(=O)O[Mg]</smiles>

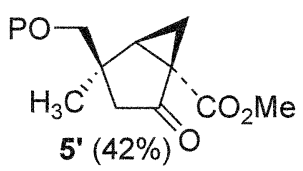
and

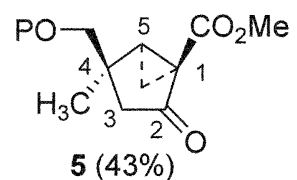

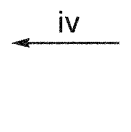<smiles>C=C[C@](C)(CC(=O)O)C(=O)C(=O)OC</smiles>

Scheme I. Construclion key bicyclo[3.1,0]hexane template. Reagenls; i) I M KOII. J:1OII. $55^{\circ} \mathrm{C}$. overnight: ii) (at) carbonyldiimidarole.

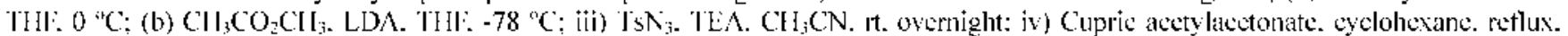
overnight.

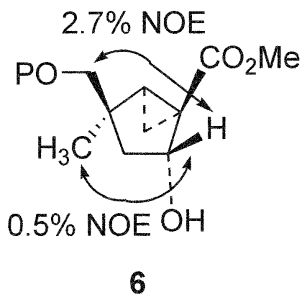

Figure 2. NOI: sludy of compound 6.

diastereofacial bias of the bicyclo[3.1,0]hexane system." The resulting hydroxyl group of compound 6 was silylated to give the ester 7 in a $92 \%$ yield. The ester 7 was sequentially subjected to hydrolysis and a Curtius reaction with diphenylphosphoryl azide in refluxing benzene to give an unstable isocyanate intermediate, which required further hydrolysis with sodium hydroxide to provide the corresponding amine 9 in a $69 \%$ two step yield. The amine derivative 9 was subjected to purine base build-up conditions using 4,6-dichloro-5-formamidopyrimidine ${ }^{12}$ in the presence of formic acid-acetic anhydride followed by the closure of the imidazole intermediate by a reaction with diethoxymethyl acetate to give the 6-chloropurine nucleoside $\mathbf{1 0}$ in a moderate yield. The deblocking of compound 10 using tetrabutylammonium fluoride and ammonolysis of resulting desilyated purine nucleoside analogue 11 was performed to give the final nucleoside 12 in a $43 \%$ two step yield. To the best of the authors' knowledge, this is the first synthetic example of a dually modified carbocyclic nucleoside with a substituent at the 4'-position. The antiviral activity of compound 12 was evaluated against various viruses including HIV-1, HSV-1, HCMV and CoxB3. However, no significant antiviral activity was observed at concentrations up to $100 \mu \mathrm{M}$ without showing any cytotoxicity to the host cell. A possible reason for the lack of activity is its unfavorable conformation for phosphorylation, which occurs during the nucleotide activation process. ${ }^{13}$

In summary, this paper reports the first synthetic example of a 4'-methyl and bicyclo[3,1.0] hexane locked nucleoside using a [3,3]-sigmatropic rearrangement, an intramolecular carbene cycloaddition reaction and a Curtius rearrangement as the key reaction steps. The extension of this strategy to the
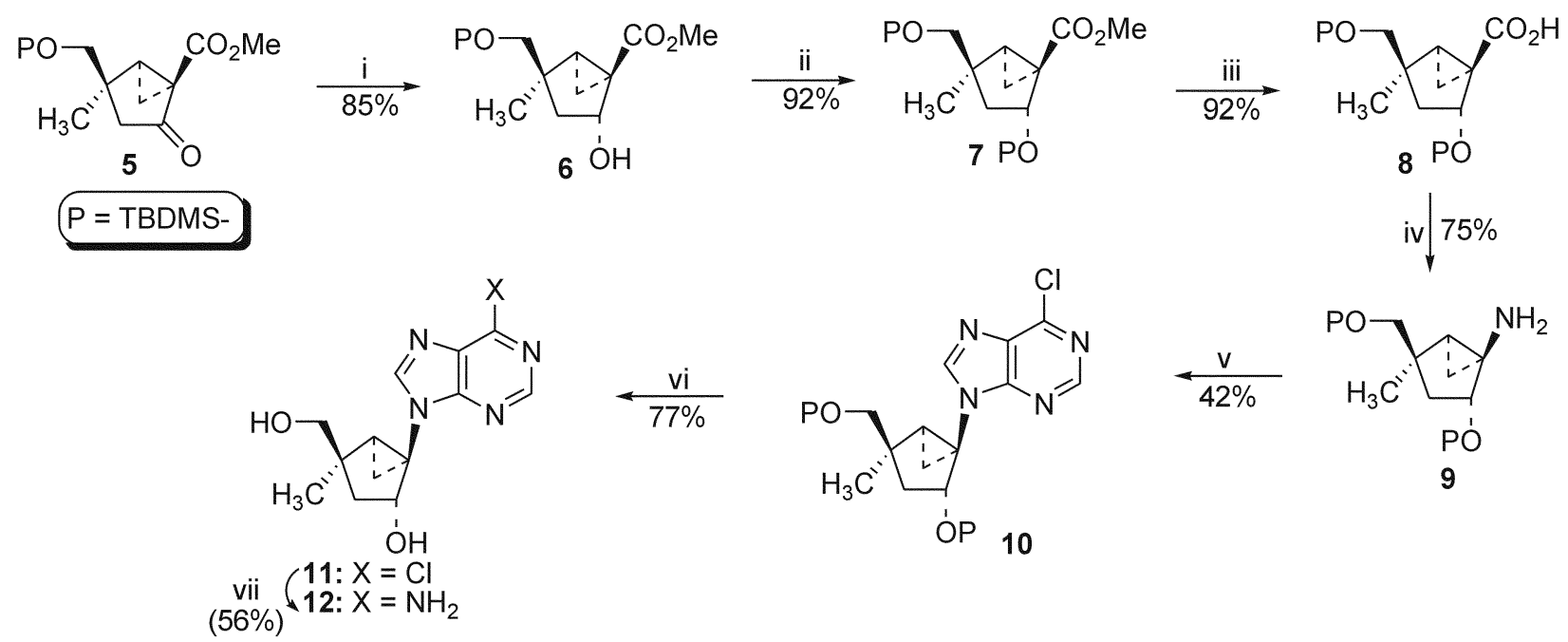

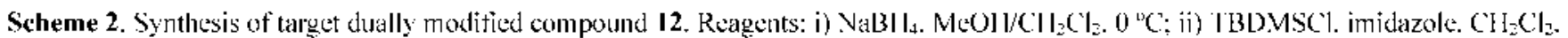
tt. $5 \mathrm{~h}$; iii) $1 \mathrm{M} \mathrm{KOH}$. EtOH. $55^{\circ} \mathrm{C}$. overnight: iv) (a) (PhO) $2 \mathrm{PON}_{3}$. TEA. benzenc. reflux; (b) NaOH. THF. rt: v) (a) 4.6-dichloro-5formamidopyrimidine. TF.A. dioxane. rellux. overnight; (b) $\mathrm{CH}_{3} \mathrm{CO}_{2} \mathrm{CH}(\mathrm{OF})_{2} .110^{\circ} \mathrm{C}$. overnight: vi) TBAF. THF. rl. 4 h: vii) $\mathrm{NH} \mathrm{H}_{3} \mathrm{NeOH}$. overnight. $90-100^{\circ} \mathrm{C}$. 
preparation of other systems, which may represent a novel type of nucleoside. is currently being investigated.

\section{Experimental Section}

All chenicals were of reagent grade and were used as purchased. All moisture-sensitive reactions were performed in an inert atmosphere of either $\mathrm{N}_{2}$ or Ar using distilled dry solvents. The elemental analyses were performed using an Elemental Analyzer System (Profile HV-3). The NMR spectra were obtained on a bruker 300 Fourier transform spectrometer and are reported in $\delta$ (ppm) downfield from tetrantetlyylsilane (TMS), used as a standard.

( \pm )-3-(tert-Butyldimethylsilyloxymethyl)-3-methyl-pent4-enoic acid (2): To a solution of conpound 1 (7.0 g. 24.4 mmol) in ethanol $(200 \mathrm{~mL})$, a $\mathrm{KOH}$ solution $(48 \mathrm{~mL} 1.0 \mathrm{M}$ solution in ethanol) was added and stirred overnight at $55^{\circ} \mathrm{C}$. After cooling the mixture to room temperature, the reaction solution was concentrated under reduced pressure. Water $(500 \mathrm{~mL})$ was poured into the residue. which was then acidified to $\mathrm{pH} 4$ with $c \cdot \mathrm{HCl}$ at $0^{\circ} \mathrm{C}$ and extracted twice with $\mathrm{CH}_{2} \mathrm{Cl}_{2}$. The combined organic extract was dried under anhydrous $\mathrm{MgSO}_{4}$ and filtered. The filtrate was concentrated under reduced pressure and the residue was purified by silica gel columin chrontatography (EtOAc/hexane. $1: 4$ ) to give the acid derivative $2(5.17 \mathrm{~g} .82 \%)$ as a colorless oil: ${ }^{1} \mathrm{H}$ NMR $\left(\mathrm{CDCl}_{3}, 300 \mathrm{MHz}\right) \delta 5.81$ (dd. $\left.J=17.0,10.2 \mathrm{~Hz}, \mathrm{IH}\right)$. $5.10(\mathrm{~d}, J=10.2 \mathrm{~Hz} . \mathrm{IH}) .5 .09$ (d. $J=17.0 \mathrm{~Hz} .1 \mathrm{H}), 3.57$ (dd. $J=13.6,8.4 \mathrm{~Hz}, 2 \mathrm{H}$ ). $2.37(\mathrm{~s}, 2 \mathrm{H}) .1 .09$ (s, 3H). $0.86(\mathrm{~s}$. $9 \mathrm{H}), 0.02(\mathrm{~s}, 6 \mathrm{H})$; Anal calc for $\mathrm{C}_{13} \mathrm{H}_{26} \mathrm{O}_{3} \mathrm{Si}: \mathrm{C}, 60.42: \mathrm{H}$. 10.14. Found: C, 60.27: H, 10.11

( \pm )-5-(tert-Butyldimethylsilyloxymethyl)-3-0x0-5-methylhept-6-enoic acid methyl ester (3): To a solution of the acid 2 (3.0 g. $11.6 \mathrm{mmol})$ in dry THF $(40 \mathrm{~mL})$, carbonyldiimidazole $(2.06 \mathrm{~g})$ was added at $0^{\circ} \mathrm{C}$. The temperature was maintained at $0^{\circ} \mathrm{C}$ for $1 \mathrm{~h}$, which was then allowed to warm to room temperature. After $2 \mathrm{~h}$. a solution of $\mathrm{LiCH}_{2} \mathrm{COOCH}_{3}$ (prepared from $\mathrm{CH}_{3} \mathrm{CO}_{2} \mathrm{CH}_{3}, \mathrm{LDA}$ ) was added to the reaction mixture at $-78^{\circ} \mathrm{C}$. Subsequently, the reaction was stirred for a further $2 \mathrm{~h}$ at $-78^{\circ} \mathrm{C}$. quenched with a $1 \mathrm{~N} \mathrm{HCl}$ solution. allowed to reach room temperature, and acidified to $\mathrm{pH} 5$ with additional $\mathrm{HCl}$. The mixture was extracted using EtOAc $(2 \times 300 \mathrm{~mL})$. The combined organic layer was dried over $\mathrm{MgSO}_{4}$. filtered, and evaporated. The residue was purified by silica gel column chromatography (EtOAc/ hexane, $\mathrm{I}: 10)$ to give compound $3(3.1 \mathrm{~g}, 85 \%)$ as a colorless oil: ${ }^{1} \mathrm{H}$ NMR $\left(\mathrm{CDCl}_{\hat{j}}, 300 \mathrm{MHz}\right) \delta 5.81$ (dd. $J=17.5$. $11.0 \mathrm{~Hz} .1 \mathrm{H}), 5.08(\mathrm{~d}, J=17.5 .11 .0 \mathrm{~Hz} . \mathrm{HH}), 3.67(\mathrm{~s}, 3 \mathrm{H})$. 3.54 (dd. $J=13.4,9.4 \mathrm{~Hz}, 2 \mathrm{H}$ ). 3.42 (s. $2 \mathrm{H}), 2.60$ (s, $2 \mathrm{H}$ ). $1.10(\mathrm{~s}, 3 \mathrm{H}) .0 .85(\mathrm{~s}, 9 \mathrm{H}) .0 .03(\mathrm{~s}, 6 \mathrm{H})$; Anal calc for $\mathrm{C}_{16} \mathrm{H}_{30} \mathrm{O}_{4} \mathrm{Si}: \mathrm{C} .61 .11 ; \mathrm{H} .9 .61$. Found: C. 60.89; H. 9.76.

(土)-5-(tert-Butyldimethylsilyloxymethyl)-2-diazo-3-oxo5-methyl-hept-6-enoic acid methyl ester (4): To a solution of compound $3(2.0 \mathrm{~g} .6 .35 \mathrm{mmol})$ in dry $\mathrm{CH}_{3} \mathrm{CN}(30 \mathrm{~mL})$. triethylanine $(2.2 \mathrm{~mL})$ and $p$-toluenesulfonyl azide $(1.25 \mathrm{~g}$. $6.35 \mathrm{mmol}$ ) was added slowly at $0^{\circ} \mathrm{C}$. The reaction mixture was allowed to warm to room temperature and stirred overnight. The reaction mixture was poured into ether and a $2 \mathrm{~N} \mathrm{NaOH}$ solution and stirred for an additional $30 \mathrm{~min}$. The organic layer was separated. dried over anhydrous $\mathrm{MgSO}_{4}$ and filtered. The filtrate was concentrated under vacuum, and the residue was purified using silica gel column cluromatography (EtOAc/hexane, $1: 20$ ) to give the diazo compound 4 (1.92 g. 89\%): ${ }^{1} \mathrm{H}$ NMR (CDCl, $\left.300 \mathrm{MHz}\right) \delta$ $5.90($ dd,$J=17.0 .10 .4 \mathrm{~Hz} . \mathrm{lH}), 5.04(\mathrm{~d} . J=10.4 \mathrm{~Hz} . \mathrm{lH})$, 5.00 (d. $J=17.0 \mathrm{~Hz} . \mathrm{lH}), 3.80(\mathrm{~s}, 3 \mathrm{H}) .3 .63(\mathrm{dd}, J=12.8,9.5$ Hz. $2 \mathrm{H}), 3.2 \mathrm{l}$ (s. $2 \mathrm{H}), 1.04$ (s. $3 \mathrm{H}) .0 .86(\mathrm{~s}, 9 \mathrm{H}) .0 .02$ (s, $6 \mathrm{H})$; Anal calc for $\mathrm{C}_{16} \mathrm{H}_{28} \mathrm{~N}_{2} \mathrm{O}_{4} \mathrm{Si}: \mathrm{C}, 56.44 ; \mathrm{H}, 8.29: \mathrm{N}, 8.23$. Found: C. $56.51 ;$ H. 8.31: N, 8.11.

(rel)-(1R,4R,5S)-4-(tert-Butyldimethylsilyloxymethyl)-2oxo-4-methyl-bicyclo[3.1.0] hexane-1-carboxylic acid methyl ester (5): To a solution of $4(5.0 \mathrm{~g}$. $14.68 \mathrm{mmol})$ in andydrous cyclohexane $(34 \mathrm{~mL})$, acetylacetonate $(3.97 \mathrm{~g}$. $14.68 \mathrm{mmol}$ ) was added at room temperature. The reaction mixture was refluxed ovemight. filtered through Celite and the solid cake was washed with EtOAc. The filtrate was concentrated under reduced pressure. The residue was purified by silica gel column chromatography (EtOAc/hexane, $1: 5$ ) to give compound 5 ( $1.97 \mathrm{~g} .43 \%$ ) and 5 ' (1.92 g. 42\%) as colorless syrup: compound 5: ${ }^{1} \mathrm{H}$ NMR $\left(\mathrm{CDCl}_{2}, 300\right.$ $\mathrm{MHz}) \delta 3.71(\mathrm{~s} .3 \mathrm{H}) .3 .55(\mathrm{~d} . J=10.8 \mathrm{~Hz} . \mathrm{HH}) .3 .32(\mathrm{~d} . J=$ $10.8 \mathrm{~Hz} . \mathrm{lH}$ ), 2.41 (dd. $J=8.4 .5 .7 \mathrm{~Hz} . \mathrm{lH}$ ), 2.05 (s. $2 \mathrm{H}$ ), $1.90(\mathrm{dd}, J=8.4 .4 .6 \mathrm{~Hz}, 1 \mathrm{H}) .1 .47(\mathrm{dd}, J=5.1 .1 .8 \mathrm{~Hz} .1 \mathrm{H})$, $1.10(\mathrm{~s} .3 \mathrm{H}), 0.84(\mathrm{~s} .9 \mathrm{H}), 0.02$ (s. $6 \mathrm{H})$. Anal calc for $\mathrm{C}_{16} \mathrm{H}_{28} \mathrm{O}_{4} \mathrm{Si}: \mathrm{C}$. 61.50: H, 9.03. Found: C. 61.34: H, 9.19. Compound 5' : ${ }^{1} \mathrm{H} \mathrm{NMR}\left(\mathrm{CDCl}_{3}, 300 \mathrm{MHz}\right) \delta 3.73$ (s. 3H), 3.53 (d. $J=12.4 \mathrm{~Hz}, 2 \mathrm{H}) .2 .45$ (dd, $J=10.6,6.7 \mathrm{~Hz} .1 \mathrm{H}$ ), $2.12(\mathrm{~s}, 2 \mathrm{H}), 1.95(\mathrm{dd}, J=10.6,5.6 \mathrm{~Hz} .1 \mathrm{H}) .1 .45(t . J=5.8$ Hz. $1 \mathrm{H}$ ). $1.1 \mathrm{l}(\mathrm{s}, 3 \mathrm{H}), 0.86$ (s. 9H). 0.04 (s, 6H): Anal calc for $\mathrm{C}_{16} \mathrm{H}_{28} \mathrm{O}_{4} \mathrm{Si}$ : C. 61.50: H, 9.03. Found: C. 61.66; H. 8.82.

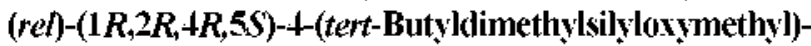
2-hydroxy-4-methyl-bicyclo[3,1.0|hexane-1-carboxylic acid methyl ester (6): A stirred solution of the bicyclic keto ester $5(5.0 \mathrm{~g} .16 .0 \mathrm{mmol})$ in a $2: 1$ mixture of $\mathrm{MeOH} / \mathrm{CH}_{2} \mathrm{Cl}_{2}$ was cooled to $0^{\circ} \mathrm{C}$ and $\mathrm{NaBH}_{4}(680.7 \mathrm{mg} .17 .9 \mathrm{mmol}$ was then added. The mixture was stirred for $1 \mathrm{~h}$ at $0^{\circ} \mathrm{C}$ and quenched by adding glacial acetic acid $(0.35 \mathrm{~mL})$. The resulting solution was concentrated and extracted using EtOAc, washed with brine, dried over $\mathrm{MgSO}_{4}$ and then filtered. The filtrate was concentrated under reduced pressure. The residue was then purified by silica gel column cluromatography (EtOAc/hexane, $1: 5$ ) to give compound 6 $(4.27 \mathrm{~g}, 85 \%)$ as a colorless syrup: ${ }^{\mathrm{H}} \mathrm{H} \mathrm{NMR}\left(\mathrm{CDCl}_{2}, 300\right.$ $\mathrm{MHz}) \delta 4.92(\mathrm{ml} . \mathrm{H}) .3 .67(\mathrm{~s}, 3 \mathrm{H}), 3.4 \mathrm{l}(\mathrm{m}, 2 \mathrm{H}), 2.20(\mathrm{~d}, J=$ $4.6 \mathrm{~Hz} . \mathrm{HH}), 1.81$ (m, $2 \mathrm{H}) .1 .25$ (dd, $J=6.0 .1 .2 \mathrm{~Hz} .2 \mathrm{H}$ ), 1.12. (s, 3H), 0.87 (s. 9H), 0.02 (s. $6 \mathrm{H}$ ); Anal calc for $\mathrm{C}_{16} \mathrm{H}_{3 i j} \mathrm{O}, \mathrm{Si}: \mathrm{C}, 61.11 ; \mathrm{H}, 9.61$. Found: $\mathrm{C}, 61.27$ : H, 9.70.

(rel)-(1R,2R,+R,5S)-2-(tert-Butyldimethylsilyloxy)-t-(tertbutyldimethylsilyloxymethyl)-+methyl-bicyclo[3.1.0]hexane1-carboxylic acid methyl ester (7): To a stirred solution of compound $6(1.0 \mathrm{~g} .3 .18 \mathrm{mmol})$ and imidazole $(429 \mathrm{mg} .6 .31$ numol) in $\mathrm{CH}_{2} \mathrm{Cl}_{2}(20 \mathrm{~mL}), t$-butyldimethylsilyl chloride (486 $\mathrm{mg}, 3.20 \mathrm{mmol}$ ) at $0^{\circ} \mathrm{C}$ was added. The mixture was stirred at room temperature for $5 \mathrm{~h}$, and quenched by adding a 
$\mathrm{NaHCO}_{3}$ solution $(5 \mathrm{~mL})$. The mixture was extracted using $\mathrm{CH}_{2} \mathrm{Cl}_{2}(200 \mathrm{~mL})$. dried over $\mathrm{MgSO}_{4}$, filtered and then concentrated. The residue was purified by silica gel column chromatography (EtOAc/hexane. $1: 15$ ) to give compound 7 $(1.25 \mathrm{~g}, 92 \%)$ as a colorless syrup: ${ }^{1} \mathrm{H}$ NMR $\left(\mathrm{CDCl}_{3} .300\right.$ MHz) $\delta 4.90(\mathrm{dd}, J=7.0 .2 .4 \mathrm{~Hz}, 1 \mathrm{H}) .3 .65(\mathrm{~s}, 3 \mathrm{H}), 3.44(\mathrm{dd}$. $J=12.5 .6 .7 \mathrm{~Hz}, 2 \mathrm{H}) .1 .75(\mathrm{dd}, J=13.5 .7 .6 \mathrm{~Hz} .1 \mathrm{H}), 1.64$ $($ dd $J=7.6 .5 .4 \mathrm{~Hz} .1 \mathrm{H}), 1.3 \mathrm{l}(\mathrm{dd}, J=7.6 .5 .0 \mathrm{~Hz}, \mathrm{lH}) .1 .21$ (dd. $J=6.4,5.7 \mathrm{~Hz} . \mathrm{lH}), 1.01(\mathrm{~s}, 3 \mathrm{H}) .0 .88(\mathrm{~m} .9 \mathrm{H}), 0.86(\mathrm{~s}$. $9 \mathrm{H}), 0.05(\mathrm{~s}, 6 \mathrm{H}),\left(0.03(\mathrm{~s}, 6 \mathrm{H})\right.$; Anal calc for $\mathrm{C}_{2 \geq} \mathrm{H}_{44} \mathrm{O}_{4} \mathrm{Si}_{2}$ : C. 61.63 ; H. 10.34 . Found $\mathrm{C}, 61.41: \mathrm{H}, 10.40$.

$(r e l)-(1 R, 2 R, 4 R, 5 S)-2$-(tert-Butyldimethylsilyloxy)-4-(tertbutyldimethylsilyloxymethyl)-4-methyl-bicyclo[3.1.0] hexane1-carboxylic acid (8): Compound 7 was prepared using the method described for synthesizing compound 2 . Yield: $92 \%$ : ${ }^{1} \mathrm{H} \mathrm{NMR}\left(\mathrm{CDCl}_{3.300 \mathrm{MHz}} \delta 4.9 \mathrm{l}(\mathrm{d} . J=7.6 \mathrm{~Hz}, \mathrm{lH}) .3 .48\right.$ $($ d. $J=8.6 \mathrm{~Hz}, 1 \mathrm{H}), 3.36(\mathrm{~d} . J=8.6 \mathrm{~Hz}, 1 \mathrm{H}), 1.8 \mathrm{l}(\mathrm{dd}, J=$ $12.6,9.8 \mathrm{~Hz}, 1 \mathrm{H}), 1.67(\mathrm{dd} . J=9.8,6.7 \mathrm{~Hz} .1 \mathrm{H}), 1.52(\mathrm{dd}, J$ $=8.7 .6 .8 \mathrm{~Hz}, \mathrm{lH}), 1.24(\mathrm{t}, J=5.8 \mathrm{~Hz} . \mathrm{lH}) .1 .07(\mathrm{~s} .3 \mathrm{H})$. $0.86(\mathrm{~s}, 18 \mathrm{H}), 0.04$ (s. $6 \mathrm{H}), 0.02$ (s. $6 \mathrm{H})$ : Anal calc for $\mathrm{C}_{21} \mathrm{H}_{42} \mathrm{O}_{4} \mathrm{Si}_{2}:$ C. 60.82; H. 10.21. Found: C, 60.66: H, 10.19.

(rel)-(1R,2R,4R,5S)-2-(tert-Butyldimethylsilyloxy)-4-(tertbutyldimethylsilyloxymethyl)-4-methyl-bicyclo[3.1.0|hex1-yl-amine (9): To a stirred solution of $8(3.0 \mathrm{~g} .7 .23 \mathrm{mmol})$ in anhydrous benzene $(50 \mathrm{~mL}) . \mathrm{Et}_{3} \mathrm{~N}(3.02 \mathrm{~mL} .21 .7 \mathrm{~nm}$ ol) and diphenylphosphoryl azide $(4.62 \mathrm{~mL} .21 .7 \mathrm{mmol})$ at $0^{\circ} \mathrm{C}$ was added. The mixture was refluxed overnight with constant stirring. cooled to room temperature and then concentrated under reduced pressure. The crude isocyanate was treated with THF $(80 \mathrm{~mL})$ and $2 \mathrm{~N} \mathrm{NaOH}(47 \mathrm{~mL})$. and then stirred for a further $30 \mathrm{~min}$ at room temperature. The resulting solution was extracted with $\mathrm{CH}_{2} \mathrm{Cl}_{2}$. dried over $\mathrm{MgSO}_{4}$. filtered and then concentrated. The residue was purified by silica gel column chromatography (EtOAC/ hexane, $1: 5)$ to give compound $9(2.09 \mathrm{~g} .75 \%)$ as a colorless syrup: ${ }^{1} \mathrm{H}$ NMR $\left(\mathrm{CDCl}_{3} .300 \mathrm{MHz}\right) \delta+.22$ (t. $J=$ $7.6 \mathrm{~Hz} .1 \mathrm{H}), 3.25(\mathrm{dd} . J=13.5 .8 .6 \mathrm{~Hz}, 2 \mathrm{H}) .1 .64(\mathrm{dd}, J=$ $13.6,8.4 \mathrm{~Hz}, \mathrm{IH}), 1.05$ (dd, $J=8.6,4.6 \mathrm{~Hz} . \mathrm{IH}) .1 .00$ (s. $3 \mathrm{H}), 0.85$ (m. $18 \mathrm{H}$ ). $0.80-0.67$ (m, $2 \mathrm{H}$ ). 0.51 (dd. $J=7.6,5.2$ $\mathrm{Hz}, \mathrm{lH}), 0.03(\mathrm{~s}, 6 \mathrm{H}), 0.01(\mathrm{~s}, 6 \mathrm{H})$ : Anal calc for $\mathrm{C}_{90} \mathrm{H}_{43} \mathrm{NO}_{2} \mathrm{Si}_{2}$ : C. 62.27: H. 11.24: N. 3.63. Found: C. 62.36: H. 11.30: N. 3.78 .

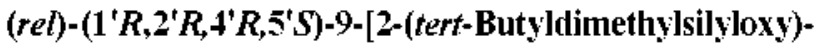
4-(tert-butyldimethylsilyloxymethyl)-t-methyl-bicyclo[3.1.0]hex-1-yl]-6-chloropurine (10): To a stirred solution of compound $9(1.0$ g. $2.59 \mathrm{mmol})$ in anhydrous 1.4-dioxane $(15 \mathrm{~mL}) . \mathrm{Et}_{3} \mathrm{~N}(1.023 \mathrm{~mL}, 7.34 \mathrm{mmol})$ and 4.6-dichloro-5formanidopyrimidine ( $498 \mathrm{mg} .2 .59 \mathrm{mmol}$ ) was added. The mixture was refluxed overnight with constant stirning. cooled to room teniperature and the resulting solid was then filtered. The filtrate was concentrated under reduced pressure and the residue was purified by silica gel column chromatography (EtOAc/hexane. $1: 2$ ) to give the acyclic imidazole intermediate as a yellow solid. This compound was immediately treated with diethoxymethyl acetate $(26 \mathrm{~mL})$ and heated oveninght at $110^{\circ} \mathrm{C}$. After cooling. the mixture was concentrated under reduced pressure. and the residue was purified by column chromatograply to give compound $\mathbf{1 0}(569 \mathrm{mg}$. $42 \%$ ) as a yellowish solid: $\mathrm{mp} 181.183{ }^{\circ} \mathrm{C}$ : ${ }^{1} \mathrm{H}$ NMR $\left(\mathrm{CDCl}_{3} .300 \mathrm{MHz}\right) \delta 8.73(\mathrm{~s}, 1 \mathrm{H}), 8.69(\mathrm{~s} . \mathrm{lH}), 4.57(\mathrm{t} . J=$ $8.2 \mathrm{~Hz}, \mathrm{lH}) .3 .72(\mathrm{~d}, J=9.0 \mathrm{~Hz}, \mathrm{lH}) .3 .45(\mathrm{~d}, J=9.0 .1 \mathrm{H})$, $1.86(\mathrm{~m}, \mathrm{lH}), 1.52(\mathrm{dd}, J=8.6,4.6 \mathrm{~Hz}, \mathrm{lH}), 1.45-1.39(\mathrm{~m}$, $3 \mathrm{H}) .1 .2 \mathrm{l}(\mathrm{s}, 3 \mathrm{H}), 0.89(\mathrm{~s}, 18 \mathrm{H}), 0.03(\mathrm{~s}, 6 \mathrm{H}), 0.0 \mathrm{l}(\mathrm{s} .6 \mathrm{H})$; Anal calc for $\mathrm{C}_{35} \mathrm{H}_{43} \mathrm{ClN}_{4} \mathrm{O}_{2} \mathrm{Si}_{2}: \mathrm{C}, 57.38 ; \mathrm{H} .8 .28 ; \mathrm{N} .10 .71$. Found: C. 57.52: H. 8.09: N, 10.54

(rel)-(1'R,2' $R, 4^{\prime} R, 5$ 'S)-9-[2-(Hydroxy)-4-(hydroxymethy])4-methyl-bicyclo[3.1.0]hex-1-yl]6-chloropurine (11): A solution of compound 10 (300 $\mathrm{mg}, 0.573 \mathrm{mmol}$ ) in THF (5 $\mathrm{mL}$ ) was treated with $1.43 \mathrm{~mL}$ of TBAF (1 M solution in THF) and stirred at room temperature for $4 \mathrm{~h}$. After concentrating the mixture, the residue was purified by silica gel colunm chromatography $\left(\mathrm{CHCl}_{3}: \mathrm{MeOH}=10: 1\right)$ to give compound $11(130 \mathrm{mg} .77 \%)$ as a white solid: $\mathrm{mp}$ 176-179 ${ }^{\circ} \mathrm{C}:{ }^{1} \mathrm{H}$ NMR (DMSO- $d_{6}$ ) $\delta 8.73$ (s. $1 \mathrm{H}$ ). 8.68 (s. $1 \mathrm{H}$ ). $4.7 \mathrm{l}$ (t. $J=6.6 \mathrm{~Hz} . \mathrm{D}_{2} \mathrm{O}$ exchangeable, $\left.\mathrm{lH}\right) .4 .45(\mathrm{~s}, \mathrm{lH}) .3 .50(\mathrm{dd} . J$ $=13.5 .8 .6 \mathrm{~Hz}, 2 \mathrm{H}) .1 .7 \mathrm{l}(\mathrm{m}, \mathrm{lH}), 1.46-1.38(\mathrm{~m}, 3 \mathrm{H}), 1.14$ (s, 3H): Anal calc for $\mathrm{C}_{13} \mathrm{H}_{13} \mathrm{ClN}_{4} \mathrm{O}_{2}: \mathrm{C}, 52.98 ; \mathrm{H}, 5.13: \mathrm{N}$. 19.01. Found: C. $52.76 ;$ H. $5.21:$ N, 18.89.

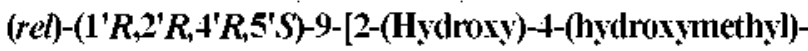
4-methyl-bicyclo[3.1.0]hex-1-yl]adenine (12): Compound 11 (200 mg. $0.678 \mathrm{mmol}$ ) was dissolved in saturated methanolic anmonia $(10 \mathrm{~mL})$ and the resulting solution was stirred overnight at $90-100^{\circ} \mathrm{C}$ in a steel bomb. After removing the reaction solvent. the yellowish residue was purified by column chromatography $\left(\mathrm{CHCl}_{2}: \mathrm{MeOH}=5: 1\right)$ to give compound 12 as a solid ( $104 \mathrm{mg} .56 \%$ ): $\mathrm{mp} 191.193^{\circ} \mathrm{C}$. UV $\left(\mathrm{H}_{2} \mathrm{O}\right) \lambda_{\max } 261.5 \mathrm{~nm}:{ }^{1} \mathrm{H}$ NMR (DMSO- $\left.d_{6}\right) \delta 8.3(\mathrm{~s}, \mathrm{lH})$, 7.96 (s. $\mathrm{lH}$ ), 7.18 (br d, $\mathrm{D}_{2} \mathrm{O}$ exchangeable, $2 \mathrm{H}$ ). 4.72 (br $\mathrm{s}$, $\mathrm{D}_{2} \mathrm{O}$ exchangeable), $4.26(\mathrm{dd} . J=8.7,6.8 \mathrm{~Hz}, \mathrm{lH}) .3 .5 \mathrm{l}(\mathrm{dd}$. $J=13.0,7.8 \mathrm{~Hz}, 2 \mathrm{H}) .1 .78(\mathrm{~m} .2 \mathrm{H}) .1 .49-1.39(\mathrm{~m}, 3 \mathrm{H}) .1 .20$ (s, lH): Anal calc for $\mathrm{C}_{13} \mathrm{H}_{17} \mathrm{~N}_{5} \mathrm{O}_{2}:$ C. $56.71:$ H. $6.22 ; \mathrm{N}$, 25.44. Found: C. $56.90 ;$ H. 6.31: N, 25.56.

\section{References}

1. (a) Marquez. V. E.: Siddiqui. M. A.: Ezzitouni. A.: Russ. P.: Wang. J. J. Med Chem. 1996. 39, 3739. (b) Marquez. V. E.: Ezzitouni. A.: Russ. P.: Siddiqui, M. A.: Ford, H.: Feldman, R. J.: Mitsuya. H.; George. C. Barchi, J. J. Jr. J. Am. Chent. Soc. 1998, 120.2780. (c) Moon. H. Y.: Ford. H. ITr: Marquez. V. E. Org. Lett. 2000. 2. 3793. (d) Choi. Y.: George. C.: Strazewski. P.: Marquez. V. E. Org Lett. 2002. 4. 589

2. Altona C.: Sundaralingam. M. J.Am. Chem. Soc. 1972. 94, 8205

3. (a) Ueland. P. M. Pharnacol Rev 1982. 31, 223, (b) Palmer, J. L. Abeles. R. H. J. Biol. Chent. 1979, 254, 1217.

4. (a) Maag. H.: Rydzewski. R. M.: McRoberts. M. I.: Verheyden1. I .P. H.: Prisbe. E. J. J. M led Chem 1992. 35. 1440. (b) Chen. M. S.: Suttmann1. R. T.: Papp. E.: Cannon. P. D.: McRobert. M. T.: Bach. C. Copeland. W. C.: Wang. T. S. F. Biochentistry 1993. 32.6002. (c) Maag. H.; Nelson. J. T.; Rios-Steiner. J. L.: Prisbe, E. J. J. Med. Chent 1994. 37, 431. (d) Maguire, A. R.: Meng. W. G.: Roberts. S. M.: Willetts. A. T. J. Chem. Soc. Perhin Trans. 11993. 1795. (e) Tenkins. I. D.: Verheyden. J. P. H.: Motfatt. J. G. J. Am. Chem. Soc. 1976. 98. 3346. (f) Guillerm. D.: Muzard. M.: Allart. B.: Guillerm, G. Bioong. Med. Chem. Lett. 1995. 5. 1455. (g) Nomura. M.: Shuto, S.; Tanaka, M.; Sasaki. T.: Mori. S.; Shigeta. S.; Matsuda A. J. Hed Chem. 1999. 22. 2901. (h) Sugimoto, I.; Shuto. S.: Mori. S.: Shigeta. S.: Matuda. A. Bioorg Ifed Chem. 
Lett. 1999.9. 385 .

5. (a) Waga. T.: Ohriu. H.: Meguro. H. Nucleosides Nucleotides 1996, 15. 287. (b) Waga. T.: Nishizaki, T.: Miyakawa J.: Ohrill. H.: Meguro. H. Biosci. Biotechol. Biochem. 1993, 57.1433.

6. Kitano K: Miura. S.: Ohrui. H.: Meguro. H. Tetrahedront 1997. 53.13315

7. (a) Yossefyeh. R. D.: Tegg. D.: Verheyden. J. P. H.: Jones. G. H.: Mottatt. J. G. Teterahedron Lett. 1992. 33. 37. (b) Youssetyeh. R. D.: Verheyden. I. P. H.: Moftàtt. I. G. J. Org. Cheme. 1979, H. 1301. (c) Jones, G. H.: Taniguchi, M.: Tegg, D.: Moffatt, I. G. J. Org. Chem. 1979. H. 1309.

8. O-Yang. C.: Kurz. W.: Eugui. E. M.: McToberts. M. J.: Verheyden. J. P. H.: Kurz. L. J.: Walker. K. A. M. Tetratedron Lett. 1992. 33. 41 .
9. (a) Hong. J. H.: Ko. O. H. Bull. Konam Chem. Soc. 2003. 24. 1284. (b) Hong. T. H. Fahak Hoeji 2003. 47.271.

10. Shin. K. J.: Moon. H. Y.: George. C.: Marquez. V. E. J. Org. Chent $2000,65,2172$.

11. (a) Grieco. P. A.: Gilman. S.: Nishizawa. M. J. Ong Chem. 1976. 11. 1485. (b) Hong. J. H. Nucleosides. Nucleotide \& Nucleic Acids 2004. 23.625.

12. Harnden. M. R.: Wyatt. P. G.: Boyd. M. R.: Sutton. D. J. Med. Chent $1990,33,187$.

13. Jurovicik, M.: Holy: A. Nicleic Acid Res. 1976, 3, 2143. (b) Spadari. S.; Maga, G.; Focher, F; Ciarrocchi. G.: Manservigi. R.; Arcamone. F.: Capobianco. M.: Careuro. A.: Colonna. F.: Lotti. S. J. Hed. Chem. 1992.35.4214. 